\title{
Espíritu Emprendedor: Reto permanente de la Academia. \\ Reflexiones sobre la experiencia del modelo de incubación de la Universidad de Pamplona.
}

HENRY MAURICIO DIEZ SILVA ${ }^{17}$

"Todo problema empequeñece ante la magnífica oportunidad que esconde tras de sí. Las historias sobre los mayores éxitos las escribieron quienes reconocieron un problema y lo convirtieron en oportunidad."

\section{RESUMEN}

El presente escrito surge de la estrecha y satisfactoria relación del autor con el modelo de incubación de la Universidad de Pamplona, y representa una herramienta de aporte a nivel de recomendaciones que buscan mejorar la efectividad de las actividades que sobre emprendimiento se llevan al interior de la Institución, y que así permitan fortalecer las experiencias exitosas y mejorar los procedimientos que presentan debilidades.

Se hace referencia al plan de negocios como una de las herramientas fundamentales para decidir la vocación emprendedora de un estudiante porque es en este donde se pueden observar los efectos de todo un proceso de emprendimiento e incubación universitario, y es allí precisamente donde al evaluar estos efectos se pueden percibir una serie de deficiencias en documentos evaluados que permiten generar las primeras conclusiones sobre algunos elementos conceptuales que se hace necesario reevaluar al interior de la academia como análisis sectoriales, análisis de mercados, planes de producción y compras.

Por último se hacen algunas recomendaciones sobre la actitud de nuestros educandos y la visión de estos ante el proceso de emprendimiento, la actividad docente, la forma de enseñar el empresarismo y los esfuerzos por promover una nueva generación de jóvenes universitarios empresarios.
Joseph Sugarman.

\section{Palabras Claves:}

Espíritu Emprendedor. Modelo de Incubación. Oportunidad. Comunidad Universitaria. 
The Free Entreprise Spirit: Permanent challenge within the Academic Society. Reflections on the experience on the Incubational Model at the University of Pamplona.

HENRY MAURICIO DIEZ SILVA ${ }^{17}$

\begin{abstract}
The present paper has grown out of the relationship the author has had with the 'incubation model' at the University of Pamplona and that represents a support tool on the level of recommendations seeking to improve the effectiveness of the activities having to do with business enterprise taking place within the institution; this permits the strengthening of successful experiences and the betterment of those procedures presenting weaknesses.

This refers to the business plan as one of the fundamental tools to decide the enterprise vocation of a student because it is here one can observe the effects of all the details involved in starting a business and this is done in the 'university incubation process'. It is here, precisely, where one can evaluate these effects and perceive a series of deficiencies found in documents already evaluated that permit the first conclusions about some of the elemental concepts which make it necessary to re-evaluate the interior of the academic aspect within the analysis of sections, analysis of markets, plans for production and buying processes.

This last aspect is to make some recommendations about the attitude of our students and the vision they have in the face of starting a business and what it entails, the professors' activity, the way to teach about enterprise and the efforts through promotion of a new generation of young people graduating from the university as businesspeople.
\end{abstract}

Keys Words:

The Spirit of Entreprise. Incubation Model. Opportunity. University community. 


\section{Espíritu Emprendedor: Reto permanente de la Academia.}

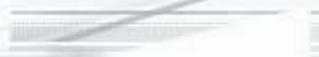

La estrecha cercanía que durante algunos años he tenido con el modelo de incubación de la Universidad de Pamplona, que ha estado representada en actividades de docencia en la Facultad de Ciencias Económicas y Empresariales, a través del Departamento de Administración, diversos procesos de asesoría a propuestas e iniciativas empresariales y planes de negocio, además de la vinculación de éstas al Fondo Emprender del SENA y otros fondos de financiación por parte de la Unidad de Emprendimiento de la Universidad; reafirmándose por medio de otro tipo de actividades académicas en la búsqueda de implementar la cultura empresarial o el espíritu emprendedor en los estudiantes de los programas adscritos a la Facultad de Ciencias Económicas y Empresariales, me han motivado aportar algunas reflexiones en referencia a la efectividad y resultados de estos procesos, y que a manera de resumen se presentan en este ensayo, esperando aportar elementos y variables considerables con miras a diagnosticar y mejorar todas las actividades emprendidas al seno de nuestra Facultad. Todo esto en razón a que garanticen a la comunidad estudiantil presente y futura, que observa detenidamente el ejemplo y eficacia que se está impartiendo, el reconocimiento de que la filosofía expuesta por los programas académicos de la nuestra Facultad es mucho más que una simple estrategia promocional universitaria y pasa a ser la entrega más tangible de opciones de vida reales y alcanzables para todos aquellos que opten por la decisión de convertir sus ideas en unidades empresariales productivas.

Si se hace referencia al término emprendedor, se encuentra que fue introducido en la bibliografía económica por Richard Cantillon en 1755 para identificar a quienes tomaban la responsabilidad de poner en marcha y llevar a término un proyecto. En sus orígenes, este término se aplicó principalmente a quienes emprendían proyectos de construcción, sobre todo construcciones de tipo militar o religioso ${ }^{18}$.

Hoy aplicamos este término a las personas que tienen una sensibilidad especial para detectar oportunidades, que poseen la capacidad de movilizar recursos para explotar esas oportunidades, y que a través de esa sensibilidad especial se hace referencia a una actitud permanente o comportamiento innato de convertir lo que se sabe 0 se reconoce en coyunturas de las que se puede tomar un beneficio financiero (desde hacer un trabajo para los compañeros de Universidad, pasando por la organización de un concierto en una ciudad, hasta la puesta en marcha de una empresa).

${ }^{18}$ NUENO, Pedro. Emprendiendo, El arte de crear empresas y sus artistas. Ediciones Deusto S.A. 2001. 
En la mayoría de programas académicos de las instituciones de Educación Superior, tanto a nivel nacional como internacional, se han reproducido programas destinados a formar emprendedores, en busca de desarrollar la capacidad de emprender, gestionar y aprovechar todas esas actividades formativas que ponen énfasis en la reflexión y el método. La técnica por excelencia, alrededor de la cual gira hoy en el mundo la formación para emprender, es el desarrollo del plan empresa (business plan), un proyecto detallado de la nueva empresa, profundizando en los aspectos más importantes de la misma $^{19}$.

En las universidades más prestigiosas se ha concluido que la mejor forma de desarrollar las capacidades relacionadas con la iniciativa o el espíritu emprendedor es orientar y encaminar a los alumnos a reflexionar sobre la elaboración de un plan de negocios. De manera paralela, influir acertadamente en los comportamientos del estudiante permite que dichos planes de negocio puedan ser llevados a la práctica con éxito. Esto se logrará con la ejecución de características como: perseverancia, espíritu de sacrificio, humildad, honradez, sensatez, cultura de resultados, trabajo en equipo interdisciplinario, pensamiento estratégico empresarial, realismo y negociación, elementos que contribuyen al aprovechamiento óptimo de las capacidades adquiridas en el aula de clase.

La Facultad de Ciencias Económicas y Empresariales desde hace algunos años se ha esforzado por implementar dentro de la estructura curricular de los programas académicos, la mentalidad empresarial y el espíritu emprendedor. Esto se evidencia mediante actividades académicas y programas del Centro Incubadora de Empresas, su unidad de emprendimiento liderada por Álvaro Parada Carvajal20.

Uno de estos programas desarrollado por la Incubadora de Empresas es la articulación de planes de negocios al Fondo Emprender del SENA. Esta actividad es un programa que promueve el Gobierno nacional, en busca de estimular la creación de nuevas empresas mediante un plan de financiación de las propuestas que se presenten y que FONADE declare como viables.

Si llegan a ser avaladas las iniciativas empresariales podrán acceder a un monto aproximado hasta 224 SMMLV no reembolsables, aportando así el capital semilla necesario para que estas nuevas empresas comiencen su etapa de operación.

La unidad de emprendimiento de la Facultad ha participado en las convocatorias del Fondo Emprender en los años 2005 - 2006, presentando más de 50 planes de negocios logrando acceder a recursos con un proyecto que en el 2006 inició sus actividades comerciales en la ciudad de Pamplona.

${ }^{19}$ NUENO, Pedro. Emprendiendo, El arte de crear empresas y sus artistas. Ediciones Deusto S.A. $2001 . p p 15-16$.

${ }^{20}$ Docente y Decano de la Facultad de Ciencias Económicas y Empresariales de la Universidad de Pamplona, premio al mejor Docente en Colombia de la cátedra virtual de emprendimiento y quien ha liderado el proceso de emprendimiento desde hace más de diez años en los programas de Administración, logrando que sea parte de la filosofía de la Universidad. 
El proceso de formulación y presentación de estos planes de negocio ante el Fondo Emprender del SENA, representa un reto para estudiantes-emprendedores y profesores-asesores, quienes en conjunto ponen a prueba sus conocimientos y competencias para formular proyectos. Estas ideas empresariales parten de un formato establecido por el SENA para tal fin, que incluye aspectos económicos, de mercado, operativos, administrativos y financieros, básicos para presentar proyectos productivos ante fondos de capital semilla o financiación.

Este proceso ha permitido que las personas vinculadas adquieran un conocimiento cada vez mayor, que se imprime en el manejo de la estructura a desarrollar desde el principio hasta la puesta en marcha de la iniciativa empresarial.

En los aspectos de mayor relevancia que se deben tener en cuenta para aceptar 0 rechazar la propuesta, se encuentran los planes de desarrollo a nivel nacional y departamental, la apuesta por cadenas productivas, las potencialidades de las regiones y la estructura detallada de los planes operativos. Así mismo, en el desarrollo de estos aspectos se evidencia una serie de vacíos desde el punto de vista académico y formativo.

Entre los temas en los que se han localizado deficiencias estructurales al formular el plan de negocios encontramos:

1. Análisis del sector al que pertenece el proyecto.

2. Análisis del mercado en el cual se piensa introducir el proyecto.

3. Estimación de los mercados potenciales y objetivos del proyecto.

4. Formulación de las estrategias de distribución, promoción y aprovisionamiento.

5. Elaboración de proyecciones de ventas de acuerdo a los análisis del mercado.

7. Elaboración de Planes de producción.

8. Estimación de Planes de compras basados en los planes de producción.

9. Estimación de la estructura de costos del proyecto.

10. Análisis y cálculo de punto de equilibrio.

11. Diseño de la estructura organizacional.

12. Elaboración de un modelo financiero coherente a la información del proyecto.

13. Evaluación financiera del proyecto de acuerdo a criterios de rentabilidad determinados (VAN, TIR, Plazo de recuperación, Beneficio/Costo).

14. Evaluación de impactos ambientales.

15. Coherencia en la estructura del proyecto y la información depositada en el plan de negocio.

16. Viabilidad técnica de los proyectos en referencia al desarrollo de las actividades propias de la cadena de valor que propone el proyecto.

Esta mención de falencias se realiza en pro de realizar aportes para mejorar la calidad académica de nuestra Facultad, en miras a construir soluciones conjuntas, traducidas en un entorno compartido y productivo, que represente un urgente alto en el camino para la academia. 
Es necesario comenzar a diseñar mecanismos efectivos de planificación y control contundentes de nuestras actividades diarias, en donde se reafirme una cultura del trabajo por resultados (proyectos asesorados, producción académica, textos leídos, material nuevo cada semestre, etc.). Además de promover el espíritu emprendedor a través de estímulos permanentes, que la interdisciplinariedad entre programas de la misma Universidad y el acompañamiento de estrategias metodológicas, se apliquen en las asignaturas logrando sacar a flote el pensamiento creativo, crítico y reflexivo propio de la comunidad universitaria, relevando el protagonismo del docente en el aula y estimulando la participación activa, calificada y permanente de los estudiantes en su propio proceso de aprendizaje.

Este proceso también reta a los docentes a pertenecer a una nueva guardia que transforme la acción didáctica-pedagógica hacia el uso intensivo de las Nuevas Tecnologías de la Información, con la inclusión de actividades prácticas que apliquen el conocimiento en la solución de problemas reales relacionados estrechamente con oportunidades de negocio vistas desde los saberes específicos de cada programa y la actualización permanente de la información que se entrega en el proceso de orientación de actividades académicas. Aquí también es importante el énfasis en el desarrollo de actitudes emprendedoras, de persistencia y de espíritu hacia la consecución de objetivos a través de ideas innovadoras y planes de negocio viables y coherentes.

No es suficiente ni debe ser satisfactorio para ninguno de nosotros sentir que la misión está cumplida cuando entregamos las evaluaciones y calificaciones a nuestros estudiantes y decidimos quiénes de estos tienen la capacidad de continuar el proceso.

Por parte de los estudiantes, el proceso ha evidenciado vacíos no sólo en la asimilación de conocimientos sino en su actitud, pasividad y cultura del mínimo esfuerzo ante el proceso. Cada estudiante debe reconocer rápidamente que el problema no es de carencia de empleo sino de exceso de la oferta de profesionales que hace que la única solución sea generar el empleo en vez de buscarlo.

Por lo tanto, es indispensable aprehender que se debe estudiar con el pensamiento de un líder y no de un subordinado, que se deben tomar las riendas de las clases para que cada ser descubra las potencialidades del conocimiento adquirido en el aula de clases y su puesta en práctica, acompañado de actualizaciones permanentes y esfuerzo compartido.

El proceso académico debe ser tan efectivo que será el mismo emprendedor quien al alistarse para culminar sus estudios deberá liderar los procesos de formulación y búsqueda de recursos de su plan de negocio. Esto se debe realizar de manera proactiva dejando las actividades logísticas y de asesoramiento para las unidades creadas para tal fin en la Universidad, como el Centro Incubadora de Empresas. 

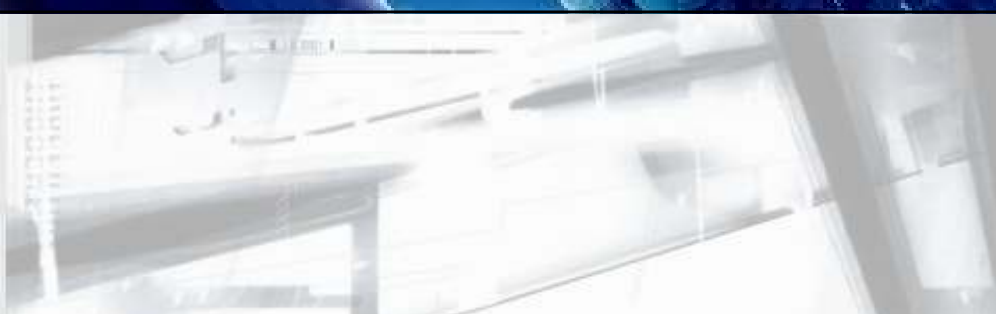

No se debe esperar que los orientadores y unidades de incubación obliguen y presionen para que haga de una idea una empresa exitosa, no sólo porque un proceso formativo "obligatorio" es más bien un proceso "deformativo" sino, sobre todo, porque si se tiene el pensamiento de que el éxito de un proyecto empresarial depende de la obtención del título que otorga al estudiante, probablemente no llegue a formarse como un profesional integral.

La comunidad universitaria que incluye docentes, estudiantes, directivos, ciudadanos y la sociedad en general, debe comprender que el espíritu emprendedor es una actitud ante la vida y un modelo mental, no una simple asignatura implantada en la estructura curricular de los programas académicos.

Es notable y evidente la brecha que existe entre el efecto de tener una idea empresarial y poseer realmente el espíritu de ponerla en práctica. Si el hecho de tener una idea interesante garantizara el tener una empresa exitosa estaríamos llenos de empresarios en todas las universidades. Pero hacer empresa implica un sinnúmero de elementos más como el ánimo, el valor y el convencimiento de traducir esa idea en una unidad productiva, a través del espíritu que contribuye a que ese mensaje promocional pase a ser una verdadera actitud ante el entorno que rodea la comunidad.

En cualquier punto en donde observemos la experiencia del modelo de incubación, encontramos que la dedicación y esfuerzo de pocos contrasta con una larga fila de individuos que hacen las cosas por salir del paso, llenos de indiferencia frente a todo lo que pasa, y frente al futuro incierto que les espera de no hacer valer el coraje comprometido con la actividad empresarial propia.

La reflexión fundamental es que no se carece de ideas, como alguna vez lo escribió Gabriel García Márquez: "Somos creativos por naturaleza". El eslabón perdido dentro de la cadena está en la ausencia del estímulo y del real espíritu emprendedor que permite ponerlas en práctica. La falta de recursos no es un elemento relevante, la realidad es que los esfuerzos hechos por desarrollar esa actitud emprendedora deben ser multiplicados para considerarlos suficientes, repensando la actividad docente para replantear las estrategias de carácter no coercitivo sino plenamente persuasivo que garanticen cambios sustanciales en la forma de pensar de nuestros profesores y estudiantes, logrando así incrementar los niveles de esfuerzo y dedicación que promuevan el reto permanente de formar los nuevos empresarios de este país. 


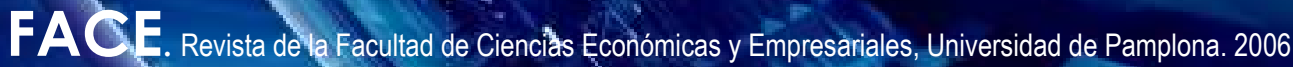

\section{CONCLUSIONES}

Una de las herramientas prácticas que permite poner a prueba la capacidad de los individuos para aprovechar las oportunidades del entorno, expresar su idea en formatos concluyentes y poner a prueba su, conocimiento y esfuerzo es el desarrollo del plan empresa ('business plan').

Para el caso de la Facultad de Ciencias Económicas de la Universidad de Pamplona la participación en el fondo emprender del SENA ha sido la mejor alternativa de carácter práctico que se ha encontrado para demostrar las verdaderas capacidades tanto de estudiantes emprendedores como de docentes asesores y poner en evidencia los vacíos y aciertos de unos y otros, del proceso formativo y de la percepción del emprendimiento, que de algún modo ha generado la inquietud en el estamento universitario de formular un modelo pedagógico mucho más robusto y sustentado, con el objetivo de formar individuos con las competencias como empresarios y profesionales preactivos. De la misma forma el proceso ha dejado al descubierto la notable y evidente brecha en la percepción equivocada que los participantes en las actividades realizadas para tal fin tienen entre el efecto de tener una idea empresarial y poseer realmente el espíritu de ponerla en práctica.

\section{BIBLIOGRAFÍA}

- CASTILLO H, Alicia. Estado del arte en la enseñanza del emprendimiento.

- COMISION EUROPEA. Guía de buenas prácticas para promover las actitudes y capacidades empresariales mediante la educación. Publicaciones DG Empresa.

- GARZON CASTRO, Hugo. El emprendimiento: Dimensión clave para el desarrollo regional (Documento Preliminar).

- Ministerio de Educación y Ministerio de Economía Chile. Descripción del Proyecto "Educación para el Emprendimiento". 